\title{
Tumor Implantation into the Intrahepatic Bile Duct after Percutaneous Ethanol Injection Therapy for Hepatocellular Carcinoma
}

\author{
Shogo Tanaka ${ }^{\mathrm{a}}$ Takatsugu Yamamoto $^{\mathrm{a}}$ \\ Nobusuke Fukumoto $^{\mathrm{b}}$ Takahiro Uenishic $^{\mathrm{c}}$ Kohichi Ohno ${ }^{\mathrm{a}}$ \\ Departments of a Surgery and ${ }^{b}$ Gastroenterology, Ishikiriseiki Hospital, \\ Higashiosaka, and 'Department of Hepato-Biliary-Pancreatic Surgery, Osaka City \\ University Graduate School of Medicine, Osaka, Japan
}

\section{Key Words}

Seeding · Thrombosis · Needle tract implantation

\begin{abstract}
A 74-year-old man who had undergone transcatheter arterial embolization for hepatitis $C$ virus-related hepatocellular carcinoma (Couinaud's segment III/IV) in April 2003 and percutaneous ethanol injection for recurrence at the same site in February 2006 was found to have dilation of the intrahepatic bile duct by computed tomography in October 2008. Contrast-enhanced computed tomography and magnetic resonance cholangiopancreatography showed a thrombosis occupying the left hepatic duct to the lateral branches with peripheral bile duct dilation. Serum concentration of alpha-fetoprotein was elevated. We performed a left hepatectomy under a preoperative diagnosis of hepatocellular carcinoma with bile duct invasion. The cut surface of the resected specimen showed a tumor thrombosis occupying the region between the left hepatic duct and lateral branches, but no tumor in the liver parenchyma. Histologic examination showed that the thrombosis in the intrahepatic bile duct was hepatocellular carcinoma. Since part of the hepatocellular carcinoma in the region treated with percutaneous ethanol injection was adjacent to the tumor thrombosis in the intrahepatic bile duct in diagnostic imaging, we diagnosed implantation into the intrahepatic bile duct due to percutaneous ethanol injection. The postoperative course was uneventful and the patient is doing well without recurrence 8 months after the operation.
\end{abstract}




\section{Introduction}

Needle tract implantation is a rare but well-documented complication following percutaneous diagnostic and therapeutic approaches such as biopsy, percutaneous ethanol injection (PEI), microwave coagulation therapy, and radiofrequency ablation for hepatocellular carcinoma (HCC). The skin, subcutaneous tissue, muscle, peritoneum, and liver parenchyma have been reported as sites of implantation [1-4], but implantation into the intrahepatic bile duct (IHBD) has not been described previously. We recently experienced a patient with tumor seeding in the IHBD after PEI.

\section{Case Report}

The patient was a 74-year-old man who had undergone transcatheter arterial embolization for hepatitis C virus-related HCC (Couinaud's segment III/IV) in April 2003 and PEI for recurrence at the same site in February 2006 (fig. 1a). The patient was found to have dilation of the IHBD by computed tomography (CT) in October 2008. Contrast-enhanced CT showed a thrombosis occupying the left hepatic duct to the lateral branches with peripheral bile duct dilation (fig. 1b). However, there was no tumor in the liver parenchyma, including the region treated by PEI. Magnetic resonance cholangiopancreatography revealed a defect of the left hepatic duct caused by the thrombosis with dilation of the peripheral lateral branches (igg. 2). Serum concentrations of carcinoembryonic antigen and carcinohydrate antigen 19-9 were within the respective reference ranges, but the serum concentration of alpha-fetoprotein was elevated $(21 \mathrm{mg} / \mathrm{ml}$; normal $<6.8 \mathrm{mg} / \mathrm{ml})$. Based on these findings, we performed a left hepatic lobectomy under a preoperative diagnosis of HCC with bile duct invasion. The cut surface of the resected specimen showed a yellowish tumor thrombosis occupying the region between the left hepatic duct and lateral branches (fig. 3 ), but no tumor in the liver parenchyma. Histologic examination showed that the thrombosis in the IHBD was HCC. Since part of the HCC in the region treated with PEI was adjacent to the tumor thrombosis in the IHBD in diagnostic imagings, we diagnosed HCC implantation into the IHBD due to PEI. The postoperative course was uneventful and the patient is doing well without recurrence 8 months after the operation.

\section{Discussion}

The frequency of needle tract implantation after PEI has been reported to be approximately $1 \%[1,2]$. During percutaneous interventional procedures, viable cancer cells that adhere to the needle can be disseminated along the needle tract during removal. In addition, blood or fluid refluxing back along the tract may carry cancer cells into the peritoneal cavity or to the surface of the skin [5]. However, some reports suggest that tumor cells disseminate within the liver rather than along the route of puncture $[3,4]$, and that needle puncture of a tumor through the portal vein may facilitate migration of tumor cells from a high-pressure tumor arterial region to the low-pressure portal vein, leading to rapid intrahepatic dissemination. In our case, the needle used for PEI might have created a pathway along its track between the tumor artery (high pressure) and the IHBD (low pressure). Intervals of 5 to 46 months between PEI and development of needle tract implantation have been reported $[1,2]$, whereas intrahepatic dissemination occurring 1 month after PEI or radiofrequency ablation has been described $[3,4]$. In our case, the interval after PEI was 32 months, which suggests that growth of the IHBD implantation may have been similar to that in needle tract implantation (route of puncture). HCC with bile duct invasion has a high risk of intrahepatic recurrence within a few months after surgery [6]. Tumor implantation into the IHBD may have different characteristics to HCC with bile duct invasion, but we are carefully observing our patient for possible recurrence. 
Fig. 1. a Contrast-enhanced CT performed before PEI in February 2006 demonstrated an intrahepatic mass (Couinaud's segment III/IV) with heterogeneous enhancement. b Contrast-enhanced CT performed 32 months after PEI (in October 2008) showed a thrombosis occupying the left hepatic duct to the lateral branches (arrow) with peripheral bile duct dilation.
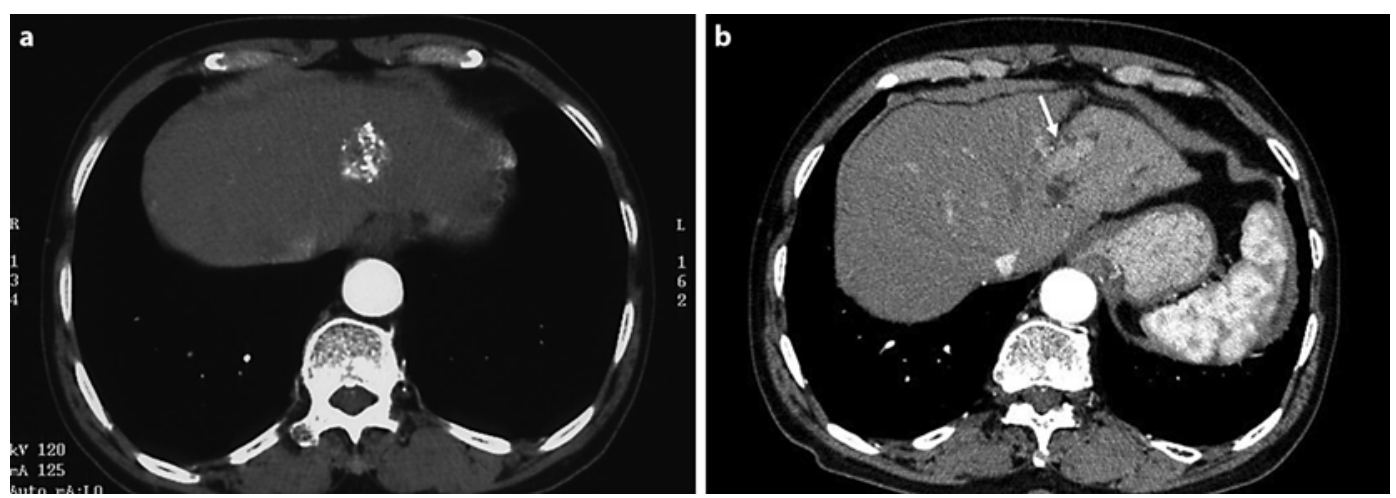

Fig. 2. Magnetic resonance cholangiopancreatography revealed a defect of the left hepatic duct and lateral branches (arrowheads) with peripheral dilation.

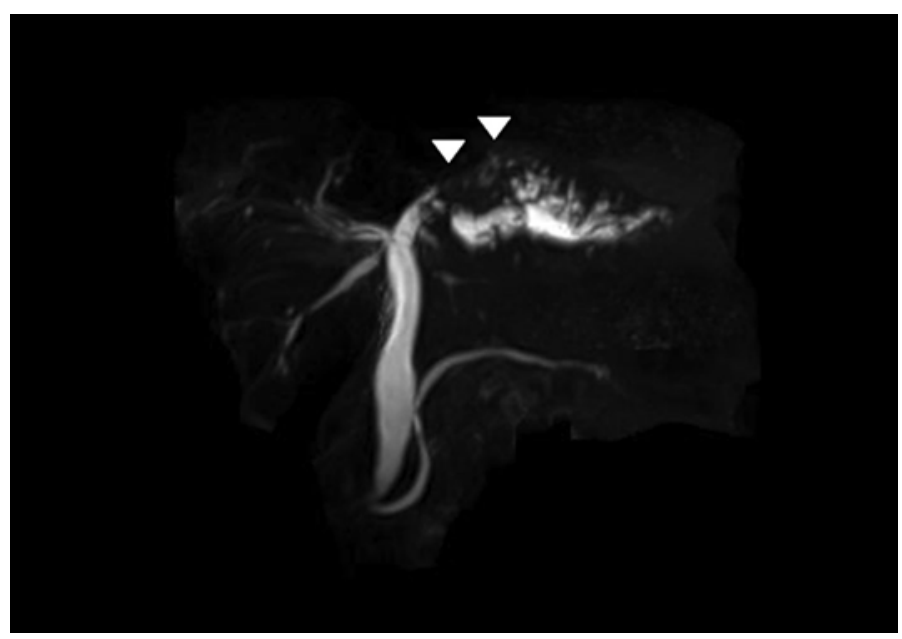


Fig. 3. The cut surface of the resected specimen showed a yellowish tumor thrombosis occupying a region between the left hepatic duct (arrow) and lateral branches (arrowheads).

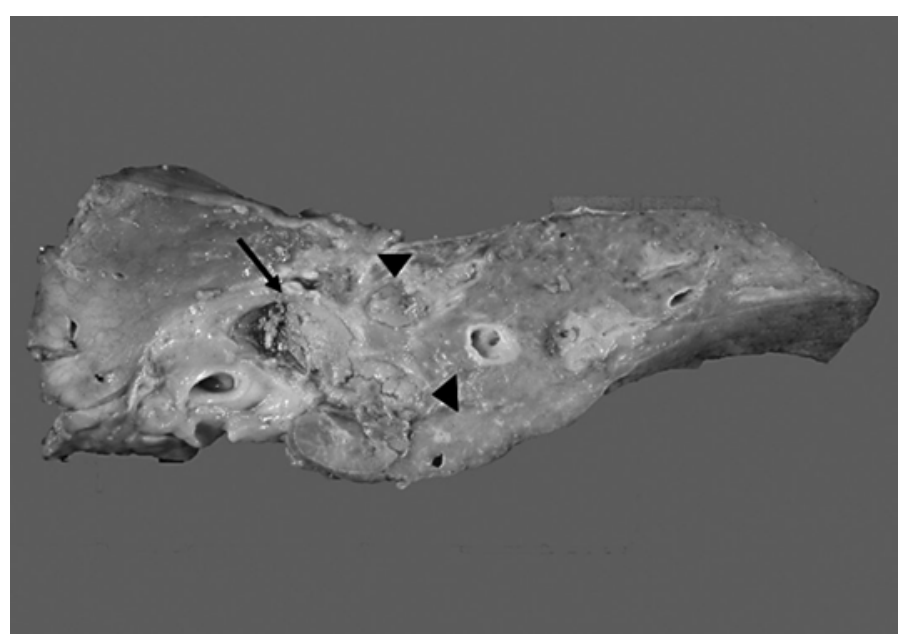




\section{References}

1 Ishii H, Okada S, Okusaka T, Yoshimori M, Nakasuka H, Shimada K, Yamasaki S, Nakanishi Y, Sakamoto M: Needle tract implantation of hepatocellular carcinoma after percutaneous ethanol injection. Cancer 1998;82:1638-1642.

2 Kosugi C, Furuse J, Ishii H, Maru Y, Yoshino M, Kinoshita T, Konishi M, Nakagohri T, Inoue K, Oda T: Needle tract implantation of hepatocellular carcinoma and pancreatic carcinoma after ultrasound-guided percutaneous puncture: clinical and pathologic characteristics and the treatment of needle tract implantation. World J Surg 2004;28:29-32.

3 Tanaka H, Ueda H, Kida Y, Fukuchi H, Ichinose M: Rapid intrahepatic tumor seeding after percutaneous ethanol injection therapy for hepatocellular carcinoma. Intern Med 2008;47:411-414.

4 Nicoli N, Casaril A, Hilal MA, Mangiante G, Marchiori L, Ciola M, Invernizzi L, Campagnaro T, Mansueto, G: A case of rapid intrahepatic dissemination of hepatocellular carcinoma after radiofrequency thermal ablation. Am J Surg 2004;188:165-167.

5 Ryd W, Hagmar B, Eriksson O: Local tumour cell seeding by fine-needle aspiration biopsy. A semiquantitative study. Acta Pathol Microbiol Immunol Scand A 1983;91:17-21.

6 Ikenaga N, Chijiiwa K, Otani K, Ohuchida J, Uchiyama S, Kondo K: Clinicopathologic characteristics of hepatocellular carcinoma with bile duct invasion. J Gastrointest Surg 2009;13:492-497. 